Novembre $27,8^{\text {h }}$ a. m. Il lato destro della grande ombra è decisamente un po' a sinistra della linea mediana della falce. $9^{\text {h }}$ a. m. Riga centrale duplice. $10^{\mathrm{b}}$ a. m. Ben visibile la linea $f$. $\operatorname{Ii}^{\mathrm{h}}$ a. $\mathrm{m}$. Lo spazio lucente in $B \mathrm{ri}$. salta bene nella relativa oscurità della regione adjacente al terminatore. $12^{\mathrm{h}}$. Nulla di mutato.

Novembre $28,5^{\text {h }}$ a. m. Notte perfetta. La grande ombra a sinistra del mezzo; duplice; sempre parallela al terminatore. Spessore tutale di essa $1 / 4$ della falce. $7^{\mathrm{h}}$ a. $\mathrm{m}$. I tre spazi lucidi $B C E$ visibili contemporaneamente. Fulgore in $A$ e $D$ nelle adjacenze dei corni. $7^{\text {h }}$ a. $\mathrm{m}$. Rivedo la diramazione $d .8^{\text {h }}$ a. m. L' ombra centrale è pallidissima. Con 7 pollici d'apertura è invisibile. Il rapporto $a b<b c$ immutato.

Da queste osservazioni risulta che nel Novembre scorso un' ombra di Venere, superiore a tutte le altre per intensità, vale a dire la striscia oscura, rimase costantemente visibile nel mezzo della falce, occupando lo spazio racchiuso fra $15^{\circ}$ e $30^{\circ}$ di distanza dal terminatore. Se il suo lato orientale passó, nel corso di un mese, dalla destra del centro alla sinistra, di qualche piccola quantità, ciò fu un puro effetto di prospettiva, dovuto all' ampliamento progressivo della fase. La distanza dell' ombra dal terminatore si mantenne invariata, e cosi pure, il parallelismo fra queste due linee non venne mai meno. Ie osservazioni di No. vembre, adunque, in pieno accordo con quelle di Luglio e

Teramo 1895 Dec. 2.

\section{Appendice. Venere nel Decembre 1895.}

In Decembre il cielo è stato qui quasi sempre coperto, e quattro sole mattine si poterono impiegare nello studio della rotazione di Venere. Esse furono Dec. 4, dalle $9^{\mathrm{h}}$ alle $11^{\mathrm{h}}$; Dec. 5 , dalle $6^{\mathrm{h}}$ alle $7^{\mathrm{h}}$; Dec. 10 , dalle $7^{\mathrm{h}}$ alle $8^{\text {h }}$; Dec. 24 , dalle $6^{\text {h }}$ alle $9^{\text {h }}$.

La grande striscia oscura non ha mostrato, in quest' intervallo, altro cambiamento che questo; essa è più lunga che in Novembre, cioè si estende un po' più di allora, tanto verso nord che verso sud. Codesta può esser una semplice apparenza, prodotta dall' aver l' ombra quasi raggiunto il diametro dei corni, guadagnando cosi una maggiore visibilità nei suoi estremi. Il parallelismo della striscia al terminatore permane tuttora. Ed è anche immutata la loro distanza. Infatti, avendo la mattina del

Teramo, 27 Decembre 1895 . di Agosto, rivelano non potersi in un mese discoprire deviamento alcuno della rotazione di Venere dalla legge di Schiaparelli. Per venire a questa conclusione mi avvalgo solo della grande ombra, che godè sempre di un alto grado di evidenza, onde la sua presenza nel mezzo della falce non potè mai revocarsi in dubbio. Le altre ombre, debolissime, e visibili solo ad intervalli, avrebbero, da sole, potuto condurre a risultati illusori. E credo pure che lo stesso avverrebbe coll' ombra maggiore, in telescopi insufficienti. Ho già detto infatti come, parecchie volte, chiuso il diaframma di Cooke fino a 7 pollici, della grande ombra non rima. nesse traccia. Perciò mi sembra che le obbiezioni mosse dal Sig. Brenner (A. N. 33 I4) al mio disegno di Agosto, e fondate sul non aver egli vista costantemente l' ombra lon. gitudinale della falce vespertina, non abbiano una base troppo solida.

La riga oscura di Agosto e' quella di Novembre si collegano probabilmente fra loro, avvolgendo Venere di una zona oscura, parallela al terminatore, e distante $30^{\circ}$ dal medesimo. Questa zona, indice, forse, di grandiosi processi atmosferici originati su Venere dall' azione solare, bisognerà tentare di rinvenirla sul disco pienamente illuminato, nella prossima congiunzione superiore. Sventuratamente ella si presenterà in troppo forte scorcio (a $0^{\mathrm{R}} .87$ dal centro) per esser un oggetto facile.

\section{Cerulli.}

24 Dec. potuto stimare, sull' immagine assai tranquilla, il rapporto dei segmenti $a b$ e $a c$, trovai $a b=4 / 10 a c$. Ora $a c$, misurato col micrometro, in parti del raggio, risultò $=1.24$ (dai dati numerici dell' Amer. Ephem. si calcolò lo stesso valore); perciò, in parti del medesimo raggio, si ebbe $a b=4 / 10 \times 1.24=0.49^{6}=\sin 29^{\circ} \cdot 7$.

La distanza, dunque, di circa $30^{\circ}$ fra $1^{\prime}$ estremità orientale della grande striscia ed il terminatore non è variata dalla fine di Ottobre a tutto Decembre, malgrado che il terminatore abbia rotato attorno al polo dell' orbita per più di $90^{\circ}$. Ciò vuol dire che sensibilmente della stessa quantità e attorno allo stesso polo han dovuto rotare anche le macchie ed il pianeta medesimo, a meno che non si voglia negare ogni legame topografico tra quelle e questo.

\title{
Ueber Veränderung einer Curve, die beobachtete Grössen darstellt, durch abschnittsweise Mittelung der Beobachtungen.
}

Bei der Berechnung der Erdaxenschwankung sind, um den Einfluss der Beobachtungsfehler herabzudrücken, aus den an den einzelnen Sternwarten erhaltenen Tagesresultaten der Schwankung innerhalb gewisser Zeitabschnitte immer Mittelwerthe verwendet worden; zum Theil wählte man die Abschnitte so, dass die Mittel nahezu gleiches Gewicht hatten, zum Theil bildete man ohne Rücksicht auf dieses die Mittel nach Monaten, also für nahezu aequidistante Epochen.
Durch diese schon mit den Tagesresultaten vorgenommene Operation wird aber eine systematische Veränderung der eigentlichen Curve bewirkt in einem Betrage, der a priori nicht sofort $z u$ ubersehen ist. Da es mindestens denkbar ist, dass aus den Dimensionen der Bahn des Erdpoles Constanten für den Erdkörper folgen, so ist es nothwendig, dies zu verfolgen.

Bei der Reduction meteorologischer Beobachtungen kommen ähnliche Erscheinungen vor und es sind mehrere 
Methoden bekannt, um derartigen Fehlern Rechnung zu tragen. Am Allgemeinsten geschieht dies, falls zur Dar. stellung der Beobachtungen eine Bessel'sche Reihe gewählt wird, so, wie im Jahre 1873 von Herrn Prof. Krueger in A. N. 1965 p. 334 angegeben worden ist. Wie sich herausstellen wird, ist in unserem Falle der Fehler irrelevant; es genügt hier, schätzungsweise zu verfahren.

Den Beobachtungen selbst nach, hat seit 1889 die Curve der Polhöhenschwankung auf einer Station in Wesentlichen den Charakter einer Sinuscurve mit der Zeit $t$ als Abscisse und den Schwankungen $y$ als Ordinaten; mitteit man dann alle Ordinaten innerhalb der Abschnitte $t=10$ bis $a, t=a$ bis $2 a, \cdots$, ordnet diesen mittleren Ordinaten die Abscissen $1 / 2 a, 3 / 2 a, \cdots z u$, so erhält man eine neue Reihe von Punkten, die innerhalb der Segmente liegen, die gebildet werden durch Sehne und Curve zwischen dem ersten und letzten Punkte jedes Abschnittes. Eine durch diese Punkte gezogene Curve liegt, da die ursprüngliche Curve $y=\sin t$ ihre concave Seite immer der Abscissenaxe zukehrt, gänzlich innerhalb der Fläche zwischen der Sinuscurve und der Abscissenaxe, sie ist flacher als jene. Eine Curve nun, die durch die neuen Punkte geht, erhält man, wenn man auf gleiche Weise alle Punkte construirt, die liegen 2 wischen $\mathrm{d} t$ bis $a+\mathrm{d} t, 2 \mathrm{~d} t$ bis $a+2 \mathrm{~d} t$, u. s. w.; für zwei beliebige, einander unendlich benachbarte, solcher Punkte $\eta, t ; \eta+\mathrm{d} \eta, t+\mathrm{d} t$ hat man dann:

Potsdam, Geodätisches Institut, 1895 November.

$$
\eta_{1}=\frac{1}{a} \int_{t-1 / 2 a}^{t+1 / 2 a} \sin t \cdot \mathrm{d} t, \quad \eta_{2}=\frac{\mathrm{I}}{a} \int_{t+\mathrm{d} t-1 / 2 a}^{t+\mathrm{d} t+1 / 2 a} \sin t \cdot \mathrm{d} t ;
$$

nach Auswerthung der Integrale erhält man:

$$
\eta_{2}-\eta_{1}=\mathrm{d} \eta=\frac{2}{a} \cdot \cos t \cdot \sin 1 / 2 a \cdot \mathrm{d} t
$$

und daraus folgt als Gleichung der neuen Curve:

$$
\eta=\frac{\sin 1 / 2 a}{1 / 2 a} \cdot \sin t
$$

Der Vergrösserungsfactor $1 / 2 a: \sin 1 / 2 a$, mit dem die $\eta$ zu multipliciren wären, um die $y$ zu erhalten, hat nun für verschiedene Verhältnisse von $a$ zur Periode $360^{\circ}$ folgende Werthe:

$\begin{array}{cccc}a & \text { Factor } & a & \text { Factor } \\ 10^{\circ} & 1+1 / 787 & 60^{\circ} & 1+1 / 21 \\ 20 & 1+1 / 196 & 70 & 1+1 / 15 \\ 30 & 1+1 / 87 & 80 & 1+1 / 12 \\ 40 & 1+1 / 49 & 90 & 1+1 / 9 \\ 50 & 1+1 / 31 & & \end{array}$

In unserem Falle, bei Verwendung von Monatsmitteln, betrüge die Vergrösserung rund ein Hundertel der ganzen Schwankung, ein Betrag, der vorläufig wenigstens ausser Betracht liegt, und keine Aenderung der bisherigen Reductionsweise in dieser Hinsicht verlangt.

Dr. R. Schumann.

\section{Beobachtungen des Cometen 1895 ... (Perrine Nov. 16)}

\begin{tabular}{|c|c|c|c|c|c|c|c|c|c|c|c|c|}
\hline 1895 & M.Z.Utrecht & $\Delta \alpha$ & $\Delta \delta$ & $V_{g l}$ & Mikr. & $\alpha$ app. & $\log p .4$ & $\delta$ app. & $\log p \cdot \Delta$ & Red.ad & pp. & * \\
\hline 18 & $17^{\mathrm{h}} 5^{2^{\mathrm{m}}} 14^{\mathrm{s}}$ & $+2^{m} 2 I^{s} !$ & $-15^{\prime} 0^{\prime \prime}$ & I. I & 2 & $13^{h} 4^{8^{m}} 11^{s} \cdot 3$ & $9.489 u$ & 704929 & & 9 & & \\
\hline 19 & 171634 & $-\circ 45.79$ & +026.9 & 12.4 & $\mathbf{T}$ & I3 $50 \quad 22.14$ & $9.524 n$ & to 1845.4 & & +1.90 & -17.0 & \\
\hline 20 & 18929 & +126.25 & +657.9 & 12.2 & $\mathbf{F}$ & 135249 & $\mathbf{I}_{\mathbf{n}}$ & -0 I5 3 & & 88 & 7.2 & \\
\hline $\begin{array}{l}25 \\
26\end{array}$ & $\begin{array}{lll}17 & 31 & 52 \\
17 & 39 & 21\end{array}$ & $\begin{array}{rr}-2 & 1.41 \\
+0 & 58.84\end{array}$ & $\begin{array}{r}-1247.5 \\
+\quad 315.9\end{array}$ & $\begin{array}{c}4 \\
2.4\end{array}$ & $\mathbf{K}$ & $\begin{array}{rrr}14 & 6 & 38.67 \\
14 & 10 & 0.00\end{array}$ & $7 n$ & $\begin{array}{lll}-3 & 28 & 36.8 \\
-4 & 14 & 41.2\end{array}$ & $\begin{array}{l}50 \\
51\end{array}$ & $\begin{array}{l}.99 \\
.02\end{array}$ & $\begin{array}{l}7.2 \\
7.2\end{array}$ & \\
\hline
\end{tabular}

am Refractor (Oeffnung $26 \mathrm{~cm}$, Brennweite $326 \mathrm{~cm}$ ) der Sternwarte in Utrecht von $A$. $A$. Nyland.

(Mitgetheilt von Herrn Prof. F. A.C. Oudemans).

\begin{tabular}{|c|c|c|c|c|c|c|c|}
\hline$*$ & $\alpha$ & $\delta$ & Autorität & $*$ & $\alpha$ & $\delta$ & Autorität \\
\hline I & I $3^{h} 45^{m} 4^{m} \cdot 3$ I & $+I^{\circ} 4^{\prime} 45^{\prime \prime} 8$ & AG. Albany 4804 & 4 & $14^{\mathrm{h}} 8^{\mathrm{m}} 3^{8.09}$ & $-3^{\circ} \times 5^{\prime} 3^{\prime \prime}$. & $M_{2} 5227$ \\
\hline 2 & 13516.03 & to 1835.5 & Rü. 4519 & 5 & 14859.14 & -4 I 39.9 & Kam 2654 \\
\hline 3 & 135121.17 & -0 22216.1 & $M_{1} 966 \mathrm{I}$ & & & & \\
\hline
\end{tabular}

Mittlere Oerter der Vergleichsterne für 1895.0.

\section{Bemerkungen.} Nov. 18 . Beobachtung von Wolken unterbrochen,
nachher wegen der Dämmerung nicht weiter fortgesetzt. Die Declination gilt für $17^{\mathrm{h}} 5^{2^{\mathrm{m}}} 14^{\mathrm{s}} \mathrm{M}$. Z., die Rectascension ist mittelst der Beobachtung von Nov. 19 von $17^{\mathrm{h}} 56^{\mathrm{m}} 9^{\mathrm{s}}$ auf diesen Moment reducirt. - Nov. 19. Der Comet hat einen ziemlich scharfen Kern von etwa 8. Grösse. Länge des Schweifes $2 \mathrm{I}^{\prime}$, Breite überall nahe $130^{\prime \prime}$; in Bezug auf eine

Utrecht 1895 Dec. 16. durch den Kern gelegte Axe (Positionswinkel, von Nord nach Ost gezählt, $329^{\circ}$ ) hat der Schweif eine symmetrische Form. -- Nov. 25 wurde ausnahmsweise ein Kreismikrometer benutzt, weil das Fadenmikrometer defect war. - Nov. 26. Det Schweif ist $40^{\prime}$ lang, $150^{\prime \prime}$ breit, und ist fast nur auf der Suldwestseite einer durch den Kern gehenden Linie (Positionswinkel $315^{\circ}$ ) sichtbar.

A. A. Nyland. 